\title{
The connected IP train and interdependent integration at the control centre
}

\author{
V. Scinteie \\ ALSTOM Transport, Montreal, Canada
}

\begin{abstract}
Through specific projects in the recent past, ALSTOM Transport has developed and integrated train-borne technologies. ALSTOM believes that the next systems challenge we face after the ongoing train-borne digital convergence developments is Interdependent Integration of information and security systems at the Control Centre. In this new context, as the central location where the field information incoming from the various subsystems is integrated and presented to the Rail Operator, the concept of Integrated Control and Security Center (ICSC) enables the Operator to manage more effectively incidents as well as to optimize response times in day-to-day operation of the Transport system.
\end{abstract}

Keywords: passenger information, infotainment, security, CCTV, control centre, train systems and rail systems.

\section{Introduction}

Today's operators face increasing demands in an increasingly complex world. Long gone are the days where passengers would accept the fact that they were on their own to figure out where they were going and leaf through a thick paper timetable to find out when they would be getting there. Today's savvy traveler takes for granted that relevant information will be readily accessible every step of their journey and demands that his/her trip be as pleasurable and safe as possible. Passengers are starting to expect being "wired" during their journey with real-time, reliable and up to date information and entertainment that they are used to get at home or at work.

In addition, in the current context of world terrorism, public transport has unfortunately become a target today for acts of violence. The tragedies in London, Madrid and Moscow confirm that rail transport is not immune to this 
danger. Operators everywhere seek solutions to minimize security risks to protect both their clientele and their assets. In this new context, the onus is on rail operators to rapidly introduce cost effective solutions which satisfy these challenging customer satisfaction and security standards.

How can a rail passenger operation meet all these great demands? They would do well to look into the communications revolution that is currently taking place around them to take advantage of these recent technological developments to offer their clients the most efficient, enjoyable and safest means of traveling. And possibly the most important recent development - and one most relevant to operators - is digital convergence.

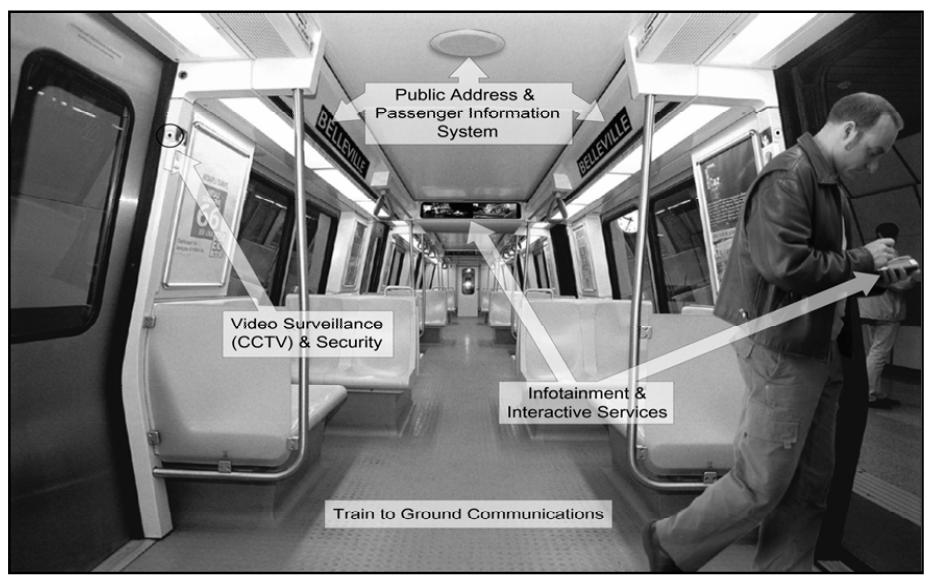

Figure 1: Today's operators are faced with the complex task of managing several different types of information including data, audio and video.

\section{What do we mean by digital convergence?}

The convergence of technology is hardly a new thing. Indeed, it can be said that it has been around for decades now. Some experts situate the beginnings of digital convergence at the RCA Pavilion at New York City's 1939 World's Fair. It was here that RCA put a new receiver on display which was a combination of television, radio, recorder, playback, facsimile and projector. This device clearly a forerunner of our present-day multifunctional devices - was years ahead of its time. In the case of this example, it was more a type of "technological convergence," since, strictly speaking, we are not dealing with digital technology, but rather analog technology. Digital Convergence can be understood as the ability to transmit audio, video and data digitally via a single network connection. This revolutionary approach has been made possible by the advent of low-cost microprocessor technology during the last 30 years or so. Although the science behind this is quite complex, the concept is simple: microprocessors have made possible the conversion of any type of signal, be it 
audio, video or data, to a series 0 s and $1 \mathrm{~s}$ (binary digits, or bits) which allows us to send formerly incompatible signals and/or multiple channels of the same type of signal down the same wire. It is therefore no longer necessary to keep these different things apart.

Many of you may still remember the huge transatlantic copper cables used for intercontinental phone communications. Why were they so big? This was simply due to the fact that each 'wire' could only handle a single analog voice 'call'. Today, because we are able to convert each signal into digital information, hundreds of voice calls can now be 'multiplexed' and sent along the same wire.

\section{Now playing in a home near you: A real-world example}

Today's average home has a telephone, cable television, an Internet connection as well as a DVD player. These services appear as typical customer "needs" in the local domestic market. A local cable television operator is currently capitalizing on system convergence by offering all of these services via the single cable connection it already has to a majority of homes in its service area. Since the operator has upgraded his network to be fully digital, he can offer analog television, digital television, video on demand, high-speed Internet, and telephone services. Customers benefit from this convergence since they not only pay less for these services as a whole, due to economies of scale, but they also only have to deal with one provider rather than two or three or four. In addition, they also get increased quality of service due to digital technology and more and better services. The cable television operator thus leverages the access he had to each home to offer multiple services, thus increasing revenue generated per household.

\section{Now playing in a train near you: Digitally converged system integration in the passenger rail industry}

In the not-too-distant past, different subsystems had to be handled separately, using different protocols, which were until recently entirely analog. Systems such as PA (Public Address), video, passenger info, communications, etc. could not use the same lines to communicate. It was physically impossible or very impractical to think of integrating these different systems, since, for example, the Passenger Information System communicated with its components in a manner which was totally incompatible with the PA system, for example. These services were also limited to basic operator voice/triggered announcements and surveillance performed solely by security personnel. Fortunately, things have changed tremendously during the last few years.

For those who think that a train digitally converged and integrated system still lies in the realm of science fiction, we have good news for you. For example, ALSTOM Passenger Information Solutions is presently implementing exactly this type of advanced system for a few of our customers. In one such project, we are deploying a digital public address system, a passenger information system, an intranet system with hundreds of access points, a retro vision and CCTV system 
with IP cameras, a passenger entertainment system in which provides movies and multimedia content on high-quality LCD screens, and an in-seat audio entertainment system, all of which are deployed on a single high-speed trainborne IP network. We are therefore not talking about hypothetical "vaporware" systems which only exist in theory. The passenger information and security systems of tomorrow are being built today.

\section{Advantages of convergence in the rail passenger industry}

\subsection{More with less}

Running five sets of cables to wire five independent subsystems costs a lot of money, takes up a lot of space and increases maintenance costs without providing any tangible advantages. Today's modern IP networks have the bandwidth necessary to provide multiple high-end services using a single Ethernet cable. What this means, paradoxically, is that an operator can offer a greater number of improved and more efficient services with less equipment in a smaller space.

\subsection{Greater and improved services}

In addition to offering their usual services, operators can offer a greater number of improved services. These can consist of a combination of any of the following: intermodal and integrated passenger information and security systems, security monitoring by exception via automated event detection \& image processing, Wi-Fi Internet, Train TV and m-Services, IP-based networks, Wireless LAN, Wireless, WAN, high-speed train LAN. It is therefore possible to offer services which were unimaginable only a few years ago.

\subsection{Reduced maintenance costs}

Since there are fewer separate hardware components as well as much less cabling, maintenance and troubleshooting costs are dramatically reduced. Imagine, if you will, a scenario where there are four different subsystems, two of which are redundant for the sake of security. This means there are six sets of controllers, cabling, power supplies, etc. all of which require regular maintenance and inspections. Now contrast this with a digitally converged and integrated system which would have only two "boxes" for controller components (one for redundancy), which communicate with all subcomponents (cameras, PA, displays, etc.) via the single high-speed IP backbone. This is a much simpler installation to inspect and maintain. Also, because there are fewer different protocols to deal with, your operations will require fewer different types of experts to service your equipment than before.

\subsection{Increased reliability}

As corollary to the above, the reduced number of power supplies, cable connections, and the equipment in general means that there is less equipment 
which can fail. In addition, the fact that all equipment communicate via the IP backbone means that it is possible to remotely monitor each element in your integrated system using a computer that is connected to the network.

\subsection{Better expansion capabilities}

Since the network used to interconnect all of the different parts of the system is based on the same standards which are used to run your office network as well as the Internet, expansion can be as easy as inserting and configuring a card in one of the available slots or connecting a new device to the Ethernet train LAN. For instance, should you wish to add additional CCTV cameras, all you need to do is install an additional IP camera and configure it so that it is recognized on the network. This is indeed a much better option than having to find space somewhere for that bulky additional controller enclosure, having to run yet another cable along your overflowing cable conduit, and adding another monitoring station and/or Operator Console Unit somewhere so that these new CCTV cameras can be monitored. Since such a digitally integrated system is based on standards accepted worldwide, you should also be able to add functionalities in the future that do not currently exist.

\subsection{Greater future-proofing}

This kind of digitally converged and integrated system is based on IP over Ethernet, the world standard for digital networking, which means that you will not only be able to service installed components for years to come but that you will be able to expand services as new ones become available. It also means that the technology upon which it is based, is not about to disappear tomorrow, unlike some other solutions based on protocols which are approaching the end of their useful lives. Solutions based on arcane, outdated and/or proprietary protocols may be a great source of headaches later on down the road when you will want to find competent technicians to service your equipment.

\subsection{Avoiding the "Christmas tree syndrome"}

The Christmas tree syndrome is what you get when you keep on adding more and more incompatible components to your system. You end up with a separate control device for each service, a spaghetti-noodle mess of wires and cables, multiple power supplies generating excess heat, numerous OCUs which must be juggled by the operator, etc. This type of installation has numerous disadvantages and almost no advantages.

\section{How do we achieve digital convergence: "The Connected IP Train"}

Digitally converged system integration is achieved by first deploying a high capacity digital communication network inside the train. As has been mentioned, an Ethernet IP backbone is the network of choice for this type of network setup. 


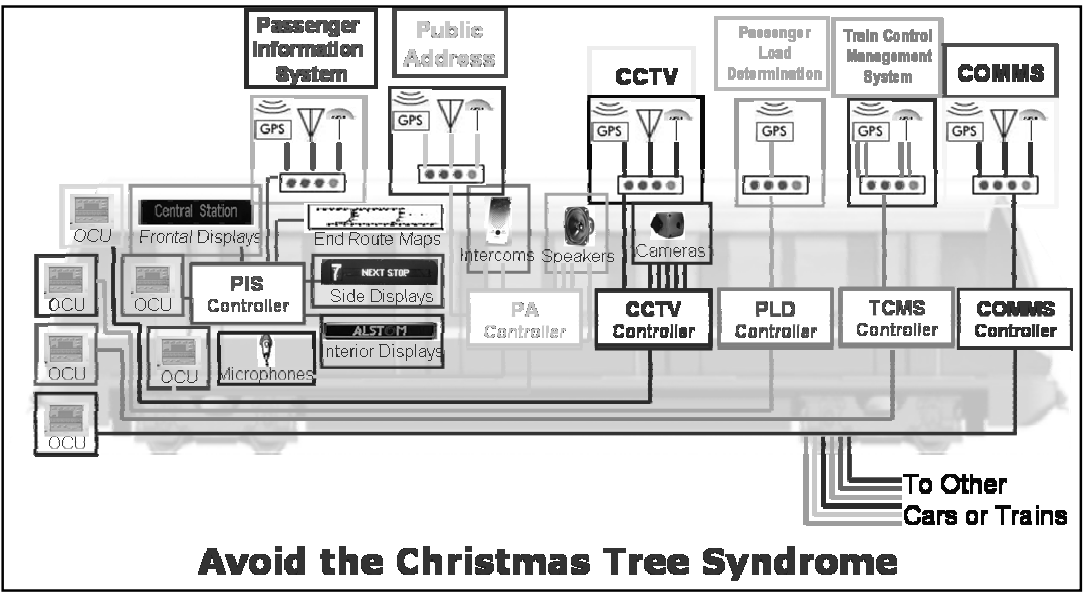

Figure 2: The more non-integrated services you add, the more your system becomes loaded like a Christmas tree.

With the IP backbone in place it's just a matter of installing controller cards (see below) in some type of 'server enclosure' that can communicate via the network IP backbone. Such components have already been developed by companies such as ALSTOM to provide numerous services such as PA, PIS, CCTV, Passenger Load Determination (PLD), Train Control Management System (TCMS) as well as many others.

In addition, because we are dealing with an integrated system, it can all be controlled using a unified Human-Machine Interface, thus greatly simplifying day-to-day operations.

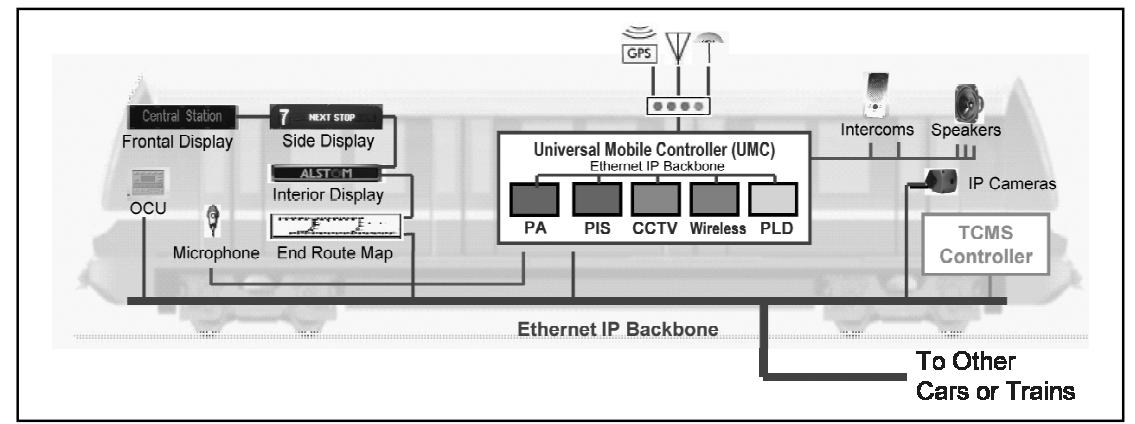

Figure 3: Digitally converged system integration is much more efficient and cost effective.

\section{The next challenge: Interdependent Systems Integration}

Once you have taken advantage of the benefits of a digitally converged system, it is important to face a new level of integration requirements. 
The train is part of a larger rail environment consisting of many other infrastructure elements such as stations (see illustration on the next page), tracks, tunnels and depots that are controlled form one or several Operation Control Centers. As the central location where the field information incoming from the various subsystems is integrated and presented to the Transport system Operator, the Operation Control Center is a critical and key element in the management of rail systems. In this context, the onus is on rail operators to deploy smart and cost effective rail systems in order to insure passenger's safety and maintain their competitiveness in the transport market. The concept of Interdependent System

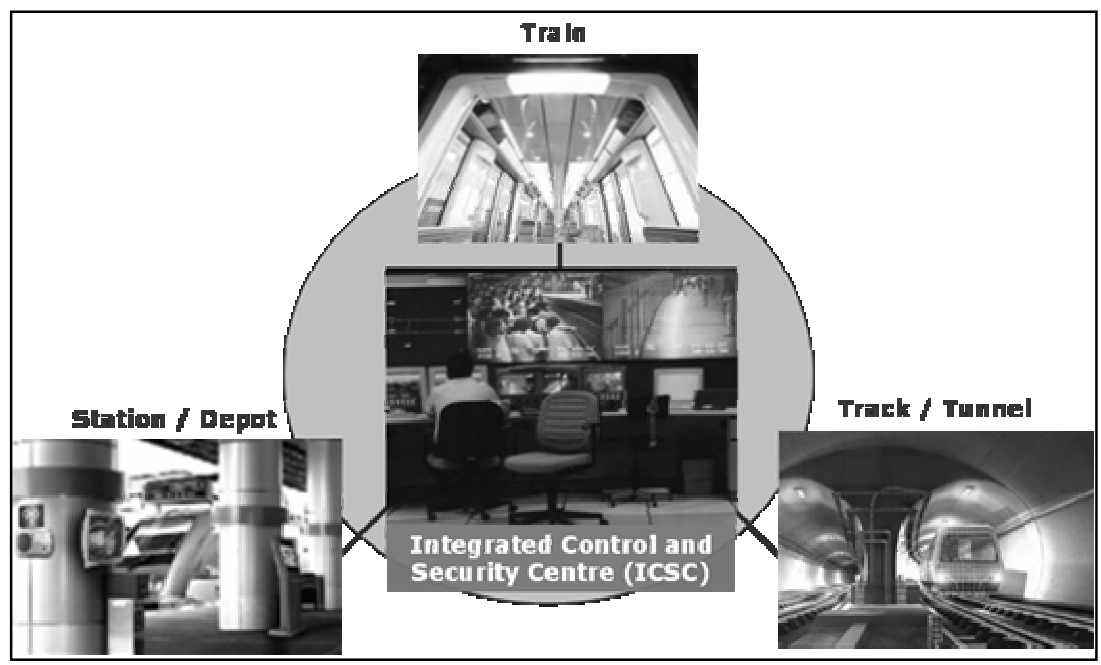

Figure 4: The train is part of a larger rail environment controlled by an Integrated Control and Security Centre (ICSC).

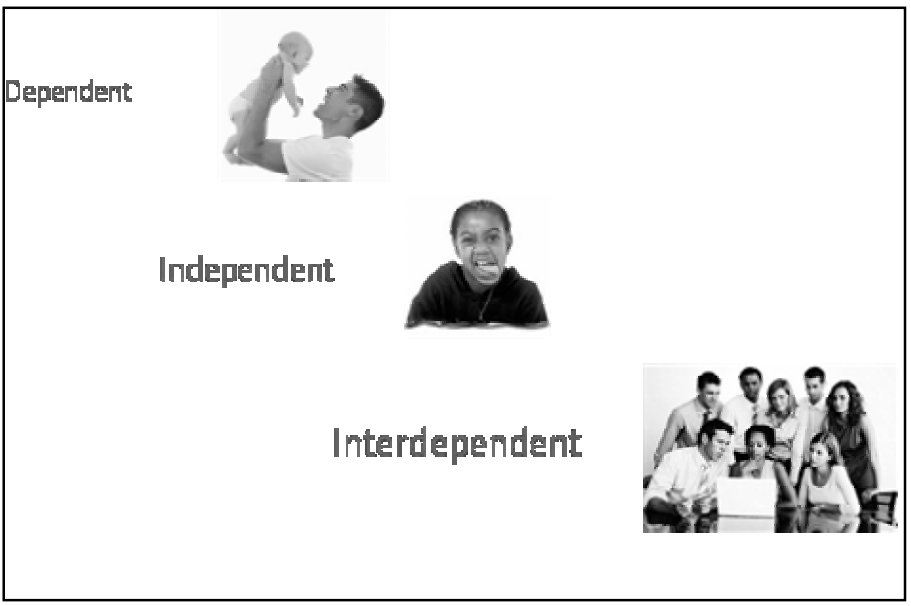

Figure 5: The road to effective integration for people in society. 
Integration with an Integrated Control and Security Center (ICSC) answers the requirement for efficient operations management and provides its full integration with the day-to-day operation of the Transport system.

\section{What is Interdependence?}

The concept of Interdependence is best illustrated by the road of effective integration of people in today's society. From early childhood through adolescence we go through different phases of dependency and independency to finally take our "whole place" in society with the many interdependent relationships necessary to carry-out in our day-to-day tasks and to live in harmony with the environment and people that surround us.

\section{How can we apply Interdependence to technology in the rail industry?}

In contemporary rail systems as in the IT (Information Technology) world we have been witnessing a spectacular evolution that is not without reminding us the road of integration for people in society. From standalone central processing with dumb slave devices, we have moved to many independent controllers all wanting to take control of the train. This anarchy provoked the unwanted "Christmas Tree Syndrome" that was described previously. To address the resulting high integration and maintenance costs as well as to improve reliability and functionally levels, ALSTOM has introduced the "Connected IP Train". This is the first level of interdependent systems integration at the train IT level. The "Connected IP Train" architecture is now a standard reference solution on all ALSTOM rolling stock platforms ranging from the Citadis trams, to metros, regional trains, intercity and the very high-speed AGV trains. The task is however not complete and there still more work do to done in terms of trainborne vital and non-vital subsystems IP integration to achieve a fully "Connected IP train".

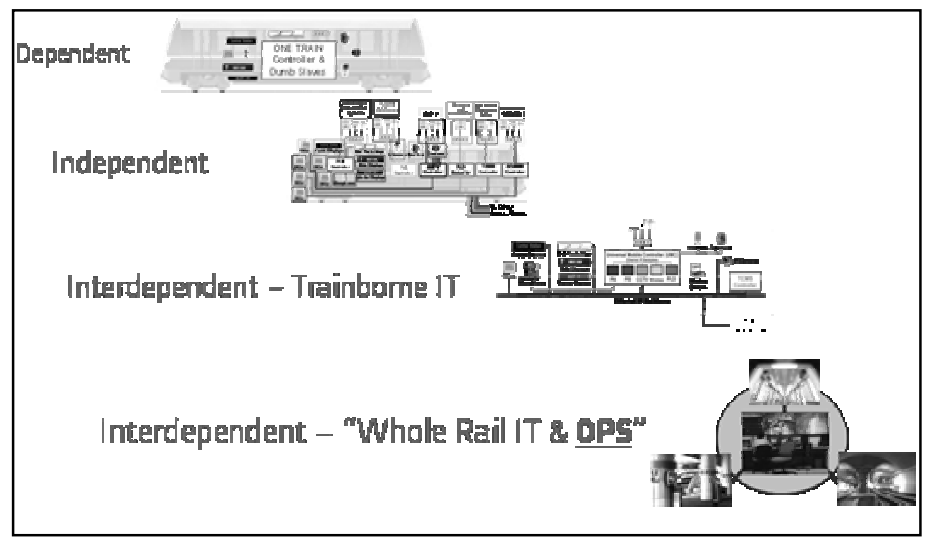

Figure 6: Interdependent Systems Integration in the rail environment. 
The next level of Interdependent Integration that several rail operators around the world started to tackle is for the larger IT rail environment including stations, depots, tracks and tunnels. To achieve rich function sets that allow rail operators to optimize operations and improve response time in emergencies, the harmonious and timely exchange of information between the various central and field IT subsystems is essential. The field information incoming from the various subsystems is integrated, arbitrated and presented to operation staff at the Integrated Control and Security Center (ICSC).

With the Integrated Control and Security Center (ICSC) approach the operator's day to day workflow is managed effectively and any abnormalities are quickly mitigated. Operating within a modern IT entrainment, the ICSC receives validated data from the multiple subsystems so that it can search for typical situations matching the patterns provided by result of the fusion of the data. The quality of information will allow for a more directive search with minimum alternatives. This allows the operator to quickly assess the situation without spending time for lengthy verification with local personnel. The system will select the reference situation from a set of typical situation for which a mitigation plan has been prepared and personnel trained for. He has the advantage of being able to react quickly to contain the abnormal situation and resume normal operations. Operator personnel will be then able to either confirm or choose the most appropriate action plan to mitigate the incident and then communicate with relevant personnel about its implementation.

\section{Conclusion}

Digital convergence is not a future promise waiting to be realized; it is here today. It is making its way into our everyday lives from our homes to the "Connected IP Trains" that bring us there. It can help passenger rail operators to offer more services, better services while at the same time reducing equipment, installation, maintenance and operating costs.

To maximize the impact of your investment it is essential however to keep in mind the "whole picture" and take advantage of Interdependent Systems Integration that enable the communication, integration, arbitration and presentation of information required to optimize operations at the ICSC. The integration of the operations, monitoring and management of security systems, train control systems, and ancillary systems is a powerful tool for the operator of modern train infrastructure. ALSTOM is currently deploying such ICSC systems that bring together multiple domains of expertise in rolling stock, rail infrastructure, telecom, intelligent sensors, analytics engines, ATS (Automated Train Supervision), SCADA, video surveillance, and safety analysis in order to meet the upcoming challenge of interdependent systems integration at the operations control centers

Although Interdependent Systems Integration can help to solve many of the problems plaguing transit operators, it also presents new challenges. If these challenges are met by a system that has been properly designed from the outset, 
796 Computers in Railways XI

then the operator can enjoy the best of both worlds: a system that is easy to use, cost efficient and future-proof while providing passengers with enhanced security, comfort and a more enjoyable ride. 